\title{
Peertechz
}

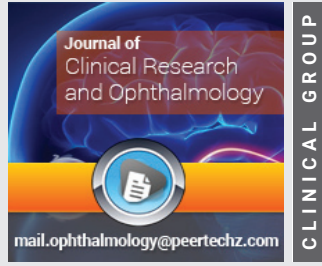

\section{From lasik to smile: Time to change laser refractive surgery}

\author{
${ }^{1} \mathrm{MD}$, OPHT, FEBOS-CR, Consultant, Vista Vision Centre, Verona, Italy \\ ${ }^{2} \mathrm{MD}$, OPHT, Chief of Hospital Ophthalmology, Bassano Del Grappa, Italy \\ ${ }^{3} \mathrm{MD}$, Ophthalmology Resident, University Eye Clinic, Parma, Italy
}

Roberto Bellucci*, Simonetta Morselli ${ }^{2}$ and Carlo Bellucci ${ }^{3}$

Check for updates

Received: 08 February, 2021

Accepted: 22 February, 2021

Published: 23 February, 2021

*Corresponding authors: Roberto Bellucci, MD, OPHT FEBOS-CR, Consultant, Vista Vision Centre, Verona, Italy, E-mail: robbell@tin.it

https://www.peertechz.com

\begin{abstract}
Purpose: To illustrate the advantages and limitations of Small Incision Lenticule Extraction (SMILE) over Laser Assisted Intrastromal Keratomileusis (LASIK) in the correction of myopia and myopic astigmatism.

Materials and methods: The two procedures are analysed and compared for the technical aspects. While LASIK directly depends on the laser efficacy to get the refractive change, SMILE only depends on the computer ability to design the refractive lenticule. The loss in precision taking place in the peripheral cornea with LASIK is not possible with SMILE. Tissue consumption and postoperative dry eye are also lower with SMILE. Procedure abortion has less consequences with SMILE. From a clinical point of view, refractive and visual results with SMILE are similar as those of Wavefront Optimized and Wavefront Guided LASIK, but re-operations are easier after LASIK than after SMILE.
\end{abstract}

Conclusion: When indicated, SMILE is better than LASIK from a technical and procedural standpoint and is equal to Wavefront Lasik as for the obtained results. LASIK is still more versatile and it is better for re-operations.

\section{Laser vision correction: LASIK and SMILE}

Laser refractive surgery was introduced in 1983, when Trokel published his famous paper about excimer laser ablation of the corneal tissue [1]. In 1990 Laser Assisted In situ Keratomileusis (LASIK) was developed by Pallikaris and Buratto [2,3] and it soon became the most popular refractive surgery procedure.

In 2010 a new technique entirely depending on the femtosecond laser, termed Small Incision Lenticule Extraction (SMILE) was conceived and applied for the first time by Sekundo [4]. At the moment SMILE is available for myopia $-.75 \mathrm{D}$ to $-10.00 \mathrm{D}$ and for astigmatism up to $3.00 \mathrm{D}$ [5].

Despite as many as 1 million procedures have been performed in 2019, SMILE is not yet widely accepted. The good results obtained with LASIK, the inability of the new procedure to correct for hyperopia and for high astigmatism, the cost of the laser, and the need to learn a new procedure are the main reasons for this delay.

As Small Incision Lenticule Extraction is transitioning from a product (single laser and company) into a procedure with two additional lasers and companies soon making it available, in this paper we will try to compare LASIK with SMILE, examining the theoretical and surgical aspects of the two procedures and the clinical results obtained over the last 10 years.

\section{Theoretical aspects}

The corneal correction of the refractive defect practically consists in the removal of a refractive lenticule from the corneal stroma. This lenticule is positive in myopia and myopic astigmatism and it is negative in hyperopia or hyperopic astigmatism. While our ability to remove the exact amount of corneal tissue in the exact location improves, we should not forget to measure the pupil diameter to avoid photopsia in night vision. In this regard SMILE makes no exception.

\section{The LASIK procedure}

In LASIK a hinged corneal flap $90-130 \mu$ thick is created either by femtosecond laser or by microkeratome (Figure 1), lifted and folded apart, and the stromal bed is remodelled by excimer laser to change the refraction of the eye. As the final step, the flap is re-positioned [6]. The safety of the surgery 
is mainly dependent on the creation of the corneal flap. Any complication at this stage will not only prevent the refractive ablation to take place, but will probably result in an impairment of the ocular condition. The flap itself can displace in the postoperative, needing replacement. Sometimes corneal folds develop eventually inducing vision deterioration [7].

The excimer laser ablates the refractive lenticule following the instructions from the laser computer and centring the ablation independently from the flap. The final result of the ablation will depend on (1) The precision of the computer modelling of the lenticule; (2) The laser/tissue interaction of each laser pulse; (3) The "cosine effect" or the loss in the pulse energy that takes place outside the corneal centre; (4) The tissue reaction during the healing phase, mainly driven by heat production. While all these aspects have been considered and improved by the laser algorithms [8] and especially by the Wavefront-Optimized (WO-LASIK) and Wavefront-Guided
(WG-LASIK), still they are the main cause of the variation in the results. Especially with high defects the obtained optical zone (free from optical aberration) may be quite smaller than the optical zone requested to the laser, with possible photic phenomena in night vision (Figure 2).

In the case of unsatisfying quantitative (refraction) or qualitative (visual quality) result it is rather easy to lift the flap and add a new excimer laser ablation, and even years after the original procedure. Topography-guided LASIK has been developed and found effective in improving vision with highly aberrated corneas [9].

\section{The SMILE procedure}

In SMILE the eye is fixed with a suction ring and connected to the laser interface at the beginning of the procedure. The refractive lenticule instructed by the laser computer is
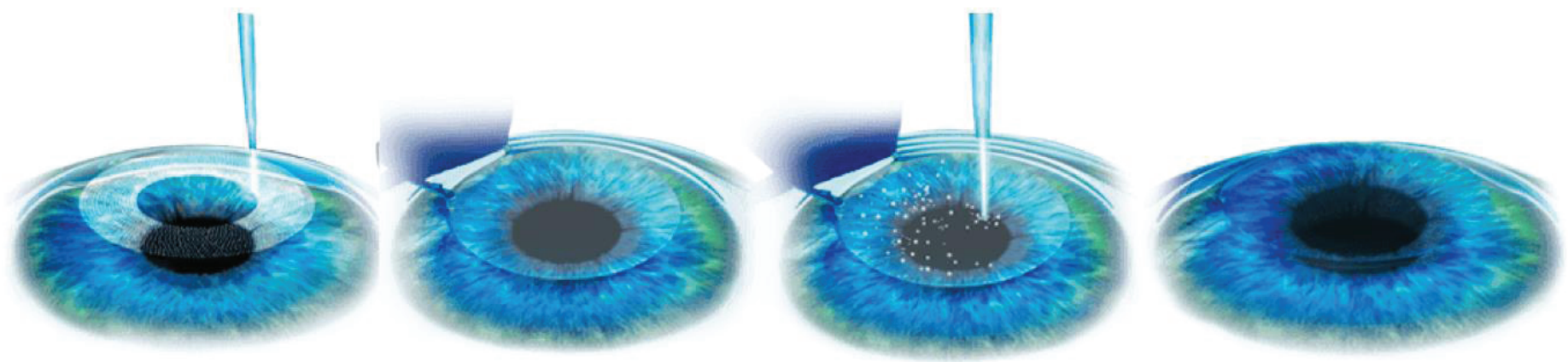

Figure 1: LASIK surgery: Femtosecond delineation of the hinged flap, flap lifting, excimer laser ablation, flap reposition.
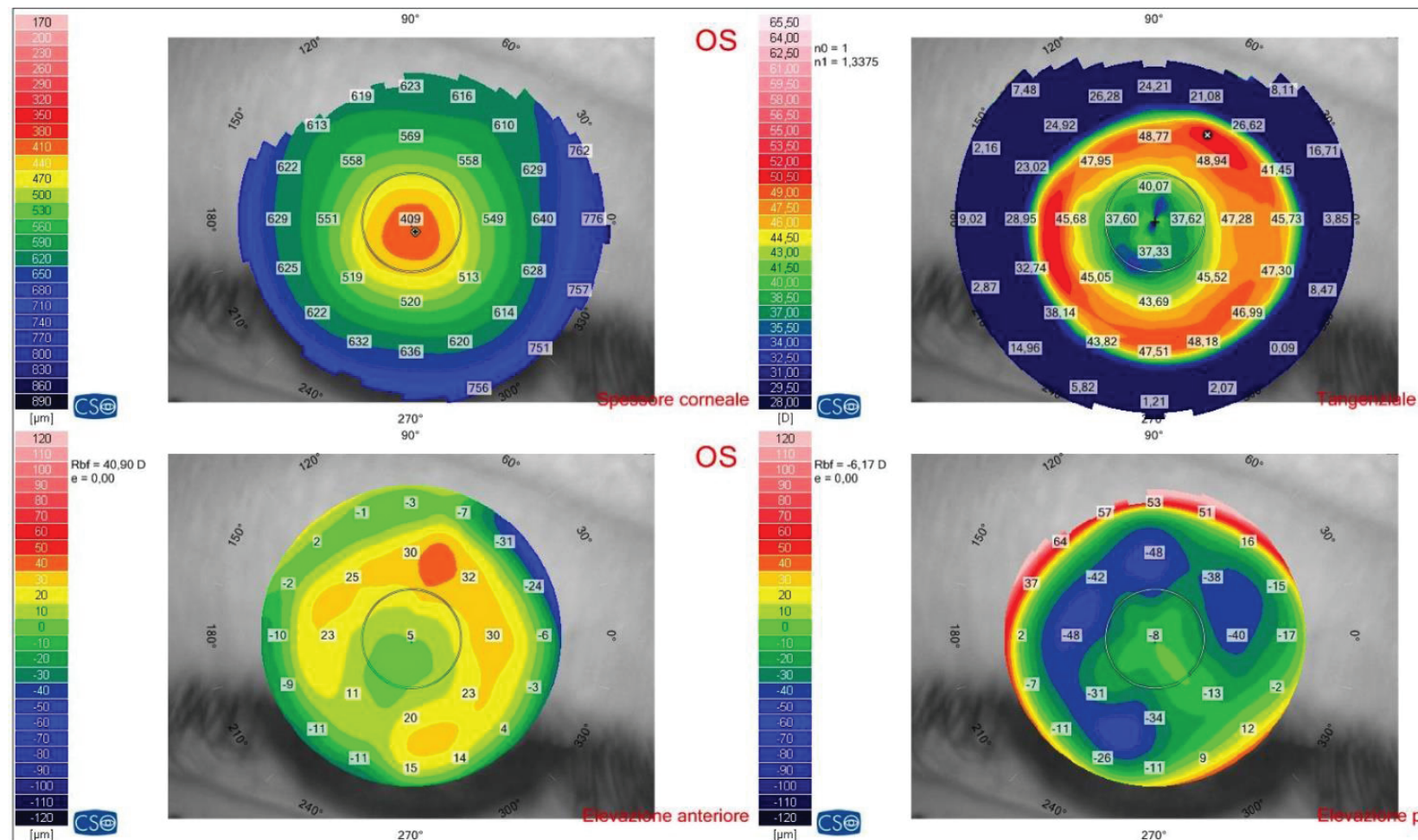

OS
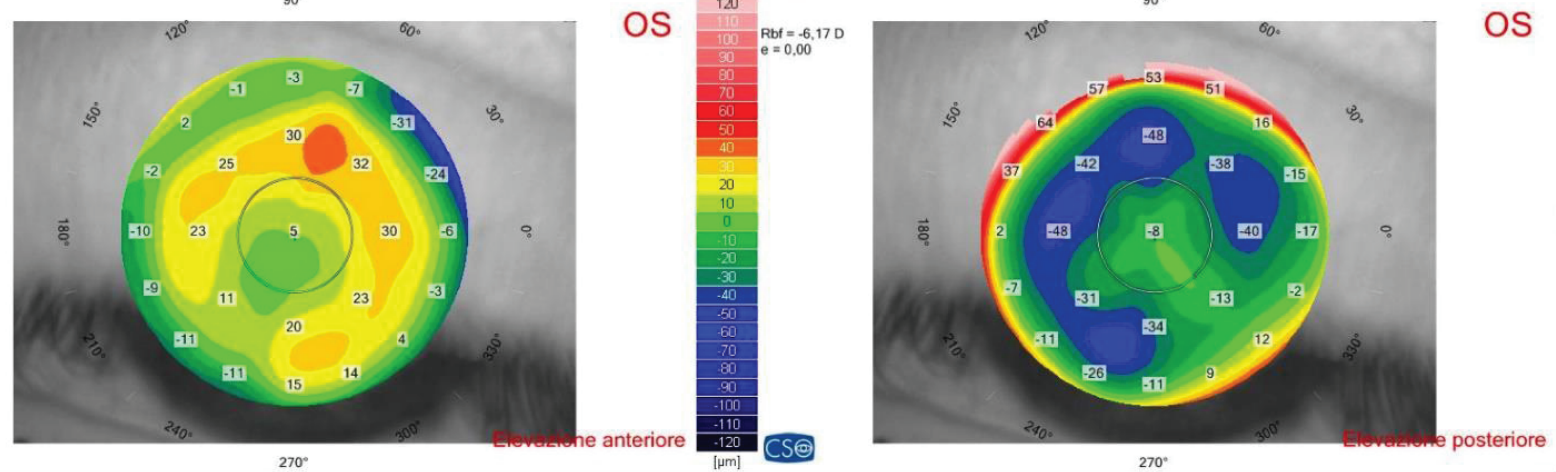

Figure 2: Result of LASIK for myopia -7.50D: Small optical zone causing night vision problems.

Citation: Bellucci R, Morselli S, Bellucci C (2021) From lasik to smile: Time to change laser refractive surgery. J Clin Res Ophthalmol 8(1): 007-011. 
designed by the femtosecond laser within the corneal stroma by creating two superimposing layers of microbubbles. Then the lenticule is dissected by the surgeon through the $2-3 \mathrm{~mm}$ incision also created by the laser, and removed from the cornea by forceps (Figure 3). The overlying cornea lays down onto the remaining corneal stroma, and a flatter corneal surface is obtained [5]. Since the incision is only $2.5-3.0 \mathrm{~mm}$ long, there is no possibility of flap displacement. Rare suction loss may abort the procedure, but the microbubbles will reabsorb and the eye will not receive any harm if no dissection is attempted. Postoperative flap folds cannot develop, however uneven microstriae are discovered at re-operations following higher myopic corrections. These microstriae have never been associated with visual problems [10].

In SMILE the femtosecond laser does not ablate the lenticule, but only designs it following the instructions of the laser computer. The possible bad laser/tissue interaction may increase the difficulty of the lenticule dissection, but the refractive result will not be affected. In this regard, we might say that in SMILE the optical result will not depend on the laser activity. The refractive lenticule will have the exact shape instructed by the computer, with no decrease of the refractive effect outside the central part of the cornea (no "cosine effect"). This results in larger optical zone as compared with most LASIK procedures, or in less tissue consumption for equal optical zone (Figure 4). However the current SMILE procedure cannot position the ablation independently from the suction ring, and any displacement of the suction ring will result in some decentration of the refractive lenticule. This (small) decentration might induce some coma in the postoperative.

In addition, no localized defect can be treated with SMILE and re-operations and enhancements can only be performed by converting the procedure into a LASIK or by PRK [11].
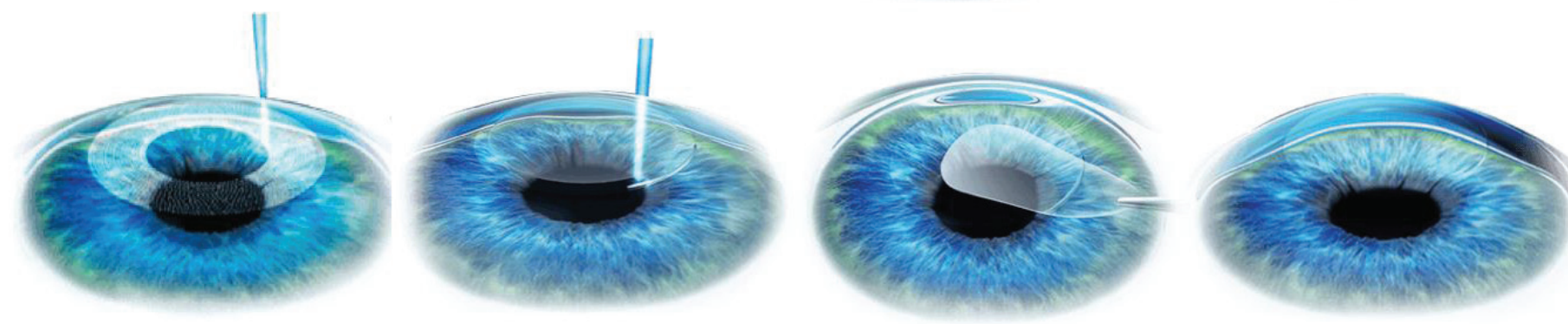

Figure 3: SMILE surgery: Femtosecond delineation of the refractive lenticule, femtosecond incision, Lenticule removal, corneal surface flattening.
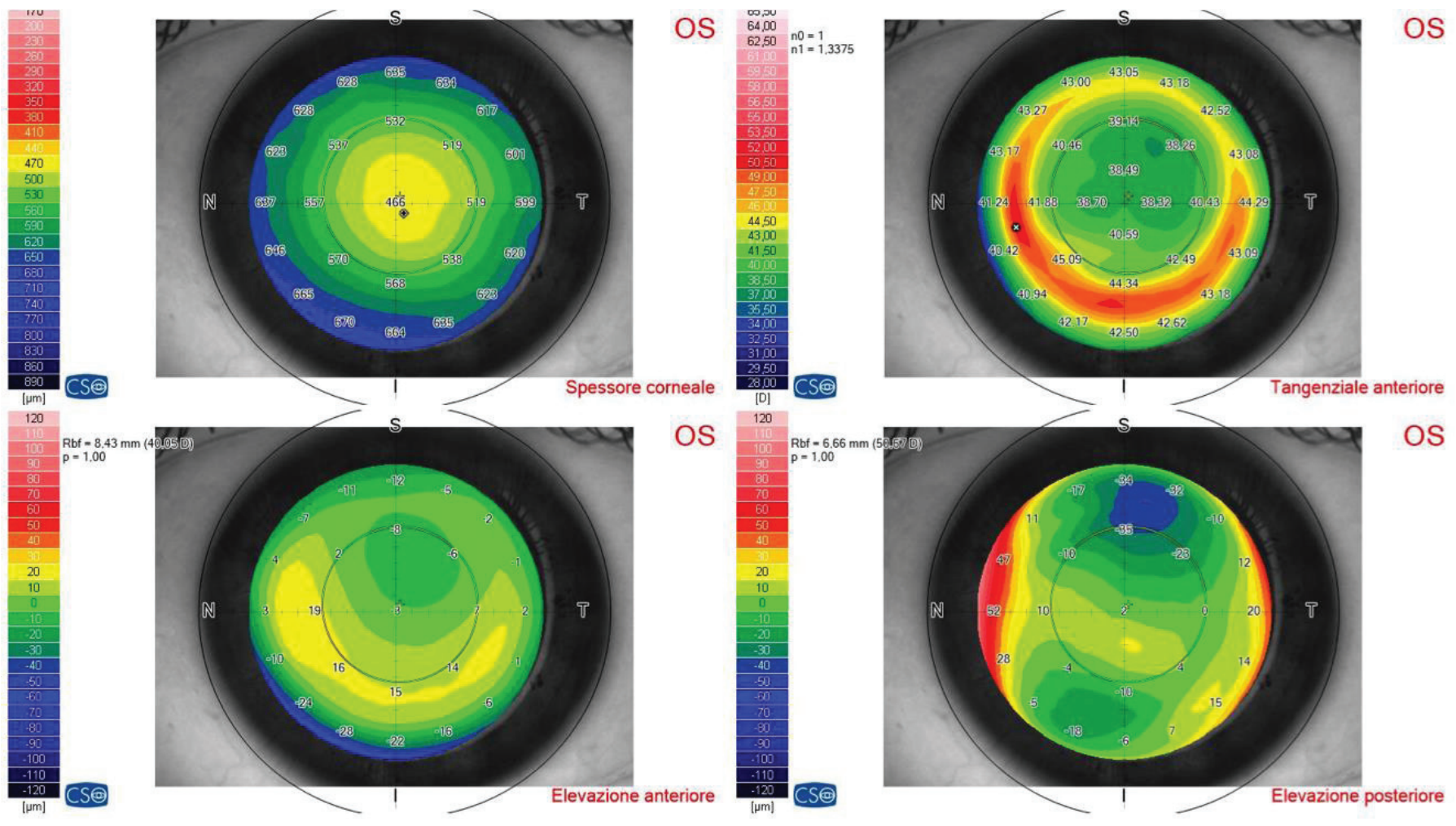

Figure 4: Result of SMILE for myopia -6.50D: Wide optical zone, no night vision problems.

Citation: Bellucci R, Morselli S, Bellucci C (2021) From lasik to smile: Time to change laser refractive surgery. J Clin Res Ophthalmol 8(1): 007-011. DOI: https://dx.doi.org/10.17352/2455-1414.000085 


\section{Clinical comparisons the two techniques}

Many studies have been published comparing the results of the two procedures. In their review published in 2016, Zhang found no difference in the efficacy (uncorrected visual acuity, refractive spherical equivalent) and in the safety (number of eyes losing one or more lines of visual acuity) of the two procedures [10]. However, the lower anatomic impairment of SMILE was followed by better corneal sensitivity and better tear film break up time in the postoperative [12]. Similar results were obtained by Breyer in his study published in 2019 [13].

The actual difference in the results between the two procedures should lie in the size of the effective optical zone, i.e. in the induction of spherical aberration. The comparison should be made with Wavefront-Optimized or with WavefrontGuided LASIK, as those procedures have been demonstrated to induce lower spherical aberration than standard LASIK [14].

Chen (2017) found lower spherical aberration with SMILE than with WG-LASIK, although with higher vertical coma [15]. Xia (2018) obtained similar results in eyes with about $8 \mathrm{D}$ of pre-operative myopia [16]. Interestingly, most authors who studied the optical aberration after SMILE and after WG-LASIK found lower corneal coma after WG-LASIK. This indicates the next evolution of the SMILE procedure should be the ability of centring the procedure after the suction ring application.

Apart from the visual results, the corneal nerve impairment is lower with SMILE with lower induction of dry eye problems in the post-operative. The possible lower impairment of the corneal biomechanics have still to be elucidated, but only 7 ectasia cases have been reported over 750.000 procedures [17]. From this point of view, currently the lower impairment of the corneal anatomy with SMILE is not considered to lower the risk for corneal ectasia, and the same safety measures we apply for LASIK are also applied for SMILE [18].

Postoperative complications are lower with SMILE than with LASIK. Flap displacement cannot occur, but rarely incomplete lenticule removal and epithelium penetration into the corneal pocket have been described $[19,20]$. The occurrence of DLK, although rare, has been reported after SMILE also [21].

\section{Discussion}

Medicine and surgery are continuously evolving, and laser refractive surgery makes no exception. After considering the technical, the surgical, and the clinical aspects of LASIK and SMILE we believe it's time to adopt the newer procedure for the correction of myopia and of myopic astigmatism. The main advantage of SMILE over LASIK can be summarized as follows:

* The micro-bubbles produced by the laser can re-absorb without impairing the eye, therefore the procedure is reversible until the lenticle dissection is initiated, and possibly even later on.

- The superficial corneal cut is much shorter than that of LASIK.

* The shape of the refractive lenticle does not depend on the laser in any way.
* The obtained optical zone is larger than in LASIK (and spherical aberration is lower).

* The tissue consumption is lower than in LASIK for the same optical zone.

* The induced heat is lower than in LASIK.

* No postoperative flap complication can occur.

There are also disadvantages that may be solved with the improvement of the procedure:

a) The centration of the procedure depends on the centration of the suction ring and is less precise than in LASIK.

b) There are two surfaces cut through microbubbles, and some light diffraction can be perceived by patients for a few weeks.

c) Re-treatments must be performed either by transforming the procedure into a LASIK or by PRK.

d) No procedure is currently available for hyperopia, simple astigmatism, myopia in excess of 10.0D, astigmatism in excess of 3.0D.

e) No wavefront-guided or topography-guided algorithms are currently available.

At the moment LASIK is still more versatile, offering the ability to treat large myopic and astigmatic errors, centration on the visual axis, and a variety of procedures tailoring specific conditions and corneas. In our opinion, LASIK can be substituted by SMILE in primary procedures when the refractive sphere is between $-0.75 \mathrm{D}$ and $-10.00 \mathrm{D}$, and the refractive cylinder is lower than 3.00D and lower than the refractive sphere [20]. The main reasons for the change are the absence of corneal flap, the lower dependence of the result from the laser beam efficiency, and the better optics of the ablated lenticule.

The future development of SMILE will probably address the current drawbacks of the procedure. The definition of precise spot energy level and spacing will produce smoother surfaces. Hyperopia treatments will soon become available. New developments of corneal transplant surgery stimulated by the SMILE experience like the SLACK technique are already on their way $[22,23]$. New femtosecond lasers with several variations of the procedure will increase scientific and technical competition thus further improving the procedure.

\section{References}

1. Trokel SL, Srinivasan R, Braren B (1983) Excimer laser surgery of the cornea. Am J Ophthalmol 96: 710-715. Link: https://bit.ly/3pGv6g6

2. Buratto L, Ferrari M, Genisi C (1993) Myopic keratomileusis with the excimer laser: one-year follow up. Refract Corneal Surg 9: 12-19. Link: https://bit.ly/3bqBAKJ

3. Pallikaris IG, Papatzanaki ME, Stathi EZ, Frenschock O, Georgiadis A (1990) Laser in situ keratomileusis. Lasers Surg Med 10: 463-468. Link: https://bit.ly/3sfT9E5

4. Sekundo W, Kunert KS, Blum M (2011) Small incision corneal 
refractive surgery using the small incision lenticule extraction (SMILE) procedure for the correction of myopia and myopic astigmatism: results of a 6 month prospective study. Br J Ophthalmol 95: 335-339. Link: https://bit.ly/2ZBiSL9

5. Reinstein DZ, Archer TJ, Gobbe M (2014) Small incision lenticule extraction (SMILE) history, fundamentals of a new refractive surgery technique and clinical outcomes. Eye Vis (Lond) 1: 3. Link: https://bit.ly/3sfNOYK

6. Bellucci R (2012) LASIK for myopia, hyperopia, and astigmatism In: Spaeth GL, Danesh-Meyer HV, Goldberg I, Kampik A: Ophthalmic Surgery. Elsevier 163-166.

7. Moshirfar M, Bennett $P$, Ronquillo $Y$ (2020) Laser in Situ Keratomileusis. 2020 Jul 31. In: StatPearls [Internet]. Treasure Island (FL): StatPearls Publishing. Link: https://bit.ly/3qMpJ04

8. Mrochen M, Hafezi F, Jankov M, Seiler T (2006) Ablation profiles in corneal laser surgery. Current and future concepts. Ophthalmologe 103: 175-183. Link: https://bit.ly/3aEZUJG

9. Ramamurthy S, Soundarya B, Sachdev GS (2020) Topographyguided treatment in regular and irregular corneas. Indian $\mathrm{J}$ Ophthalmol 68: 2699-2704. Link: https://bit.ly/3kbtr0X

10. Moshirfar M, Somani SN, Patel BC (2020) Small Incision Lenticule Extraction. In: StatPearls [Internet]. Treasure Island (FL): StatPearls Publishing.

11. Meyer B, Kunert KS (2017) SMILE: Re-Treatment Options Techniques and Results. Klin Monbl Augenheilkd 234: 98-101. Link: https://bit.ly/2NI3qtP

12. Zhang Y, Shen Q, Jia Y, Zhou D, Zhou J (2016) Clinical Outcomes of SMILE and FS-LASIK Used to Treat Myopia: A Meta-analysis. J Refract Surg 32: 256-265. Link: https://bit.ly/3k9xiva

13. Breyer DRH, Beckers L, Hagen P, Kaymak H, Klabe K, et al. (2019) Comparison of Long-term Results with Small Incision Refractive Lenticule Extraction (ReLEX SMILE) vs. Femto-LASIK. Klin Monbl Augenheilkd 236: 1201-1207. Link: https://bit.ly/2MinJhe

14. Stonecipher K, Parrish J, Stonecipher M (2018) Comparing wavefront-optimized, wavefront-guided and topography-guided laser vision correction: clinical outcomes using an objective decision tree. Curr Opin Ophthalmol 29: 277-285. Link: https://bit.ly/3qHbUjF
15. Chen X, Wang Y, Zhang J, Yang SN, Li X, et al. (2017) Comparison of ocular higher-order aberrations after SMILE and Wavefront-guided Femtosecond LASIK for myopia. BMC Ophthalmol 17: 42. Link: https://bit.ly/2Nm0ZgS

16. Xia LK, Ma J, Liu HN, Shi C, Huang Q (2018) Three-year results of small incision lenticule extraction and wavefront-guided femtosecond laser-assisted laser in situ keratomileusis for correction of high myopia and myopic astigmatism. Int J Ophthalmol 11: 470-477. Link: https://bit.ly/2OZxv8W

17. Ganesh S, Brar S, Pawar A (2017) Matched population comparison of visual outcomes and patient satisfaction between 3 modalities for the correction of low to moderate myopic astigmatism. Clin Ophthalmol 11: 1253-1263. Link: https://bit.ly/3sfAxEb

18. Damgaard IB, Reffat M, Hjortdal J (2018) Review of Corneal Biomechanical Properties Following LASIK and SMILE for Myopia and Myopic Astigmatism. Open Ophthalmol J 12: 164-174. Link: https://bit.ly/2NvkDXJ

19. Krueger RR, Meister CS (2018) A review of small incision lenticule extraction complications. Curr Opin Ophthalmol 29: 292-298. Link: https://bit.ly/2Mf6rS4

20. Shah R (2019) History and Results; Indications and Contraindications of SMILE Compared With LASIK. Asia Pac J Ophthalmol (Phila) 8: 371-376. Link: https://bit.ly/2Nwg8vQ

21. Reinstein DZ, Stuart AJ, Vida RS, Archer TJ, Carp GI (2018) Incidence and Outcomes of Sterile Multifocal Inflammatory Keratitis and Diffuse Lamellar Keratitis After SMILE. J Refract Surg 34: 751-759. Link: https://bit.ly/2MaTmZS

22. Nubile M, Salgari N, Mehta JS, Calienno R, Erroi E, et al. (2021) Epithelial and stromal remodelling following femtosecond laser-assisted stromal lenticule addition keratoplasty (SLAK) for keratoconus. Sci Rep 11: 2293. Link: https://go.nature. com/3pPx7Xr

23. Riau AK, Htoon HM, Alió Del Barrio JL, Nubile M, El Zarif M, et al. (2021) Femtosecond laser-assisted stromal keratophakia for keratoconus: A systemic review and meta-analysis. Int Ophthalmol. Link: https://bit.ly/2ZCTxAm 